\title{
Adaptive Sliding Mode Control of Mobile Manipulators with Markovian Switching Joints
}

\author{
Liang Ding, Haibo Gao, Kerui Xia, Zhen Liu, \\ Jianguo Tao, and Yiqun Liu
}

State Key Laboratory of Robotics and System, Harbin Institute of Technology, Harbin 150080, China

Correspondence should be addressed to Liang Ding, liangding@hit.edu.cn

Received 22 February 2012; Accepted 6 April 2012

Academic Editor: Xianxia Zhang

Copyright (C) 2012 Liang Ding et al. This is an open access article distributed under the Creative Commons Attribution License, which permits unrestricted use, distribution, and reproduction in any medium, provided the original work is properly cited.

The hybrid joints of manipulators can be switched to either active (actuated) or passive (underactuated) mode as needed. Consider the property of hybrid joints, the system switches stochastically between active and passive systems, and the dynamics of the jump system cannot stay on each trajectory errors region of subsystems forever; therefore, it is difficult to determine whether the closed-loop system is stochastically stable. In this paper, we consider stochastic stability and sliding mode control for mobile manipulators using stochastic jumps switching joints. Adaptive parameter techniques are adopted to cope with the effect of Markovian switching and nonlinear dynamics uncertainty and follow the desired trajectory for wheeled mobile manipulators. The resulting closed-loop system is bounded in probability and the effect due to the external disturbance on the tracking errors can be attenuated to any preassigned level. It has been shown that the adaptive control problem for the Markovian jump nonlinear systems is solvable if a set of coupled linear matrix inequalities (LMIs) have solutions. Finally, a numerical example is given to show the potential of the proposed techniques.

\section{Introduction}

The hybrid joint shown in Figure 1 was first proposed in [1-5], which is with one clutch and one brake. When the clutch is released, the link is free, and the passive link is directly controlled by the dynamic coupling of mobile manipulators; when it is on, the joint is actuated by the motor. Moreover, the passive link can be locked by the brake embedded in the joint as needed. The robot with hybrid joints is called the hybrid actuated robot.

One of the advantages of using hybrid actuated robots is that they may consume less energy than the fully-actuated ones. For example, hyperredundant robots, such as snake-like robots or multilegged mobile robots [6], need large redundancy for dexterity and specific task 


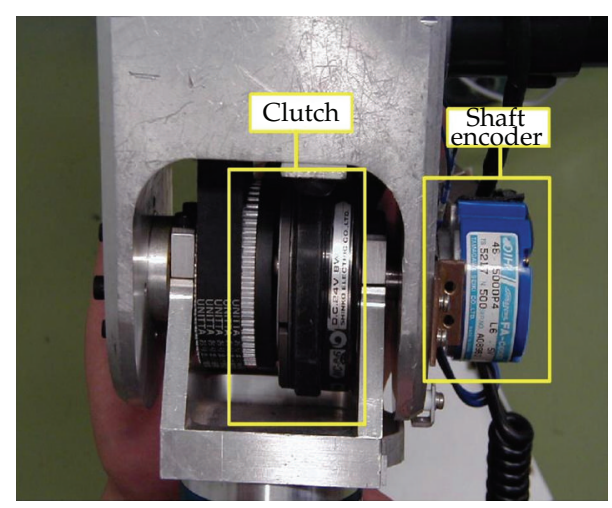

Figure 1: The hybrid joint.

completion while underactuation structure allows a more compact design and much simpler control and communication schemes. The hybrid actuated robot concept is also useful for the reliability or fault-tolerant design of fully-actuated robots working in hazardous areas or with dangerous materials. If any of the joint actuators of such a device fails, one degree of freedom of the system would be lost. It is important, in these cases, that the passive (failed) joint can still be controlled via the dynamic coupling with the active ones, so the system can still make use of all of its degrees of freedom originally planned.

Hybrid actuated mobile manipulator is the robot manipulator consisting of hybrid joints mounting on a wheeled mobile robot, which first appeared in [1-5]. Hybrid actuated mobile manipulators are different from full-actuated mobile manipulators in [7-26], due to simultaneously integrating both kinematic constraints and dynamic constraints. For these reasons, increasing effort needs to be made towards control design that guarantee stability and robustness for hybrid actuated mobile manipulators with the consideration of joint switching. The hybrid joint is also with the characteristic of underactuated the joints [27$34]$, for example, the hybrid joints in the free mode, which can rotate freely, can be indirectly driven by the effect of the dynamic coupling between the active and passive joints. The zero torque at the hybrid joints results in a second-order nonholonomic constraint $[35,36]$.

The mobile manipulator using Markovian switching hybrid joint can be loosely defined as a system that involves the interaction of both discrete events (represented by finite automata) and continuous-time dynamics (represented by differential equations). The joint switching seems to be stochastic and the switching may appear in any joints of the robot which need to develop Markovian jump linear system (MJLS) [37] to incorporate abrupt changes in the joints of mobile manipulators and use the Markovian jumping systems to guarantee the stochastic stability. Therefore, the discrete part (switching part) can be regarded as a continuous-time Markov process representing the modes of the system and the continuous part represents the dynamics state of the system, which evolves according to the differential dynamic equation when the mode is fixed. The hybrid formulation provides a powerful framework for modeling and analyzing the systems subject to abrupt joint switching variations, which are partly due to the inherently vulnerability to abrupt changes caused by component failures, sudden environmental disturbances, abrupt variation of the operation point of mobile manipulator, and so on.

The joint switching seems to be stochastic and the switching may appear in any joints of the robot, while simple switching approach cannot handle all the possibility. In this paper, 
to avoid the necessity of stopping the robot as the joint switches, MJLS method used to model and analyze switching robotic systems is an effective but challenging work.

To our best knowledge, there are few works considering MJLS method used to model and analyze switching robotic systems. In this paper, we consider the problem of adaptive control for stochastic jump systems with matched uncertainties and disturbances. The jumping parameters are treated as continuous-time discrete-state Markov process. Note that adaptive control method is one of the most popular techniques of nonlinear control design [8]. However, adaptive control for stochastic nonlinear mechanical dynamics systems with Markovian switching has received relatively little attention. Therefore, this paper will be concerned with the design of adaptive control for mobile manipulators using Markovian switching joints. There exist parameter uncertainties, nonlinearities, and external disturbance in the systems and environments under consideration. First, we design a reduced model for the wheeled mobile manipulator with switching joints. After introducing continuous-time Markov chain, adaptive control is adopted to cope with the effect of Markovian switching and nonlinear dynamics uncertainty and drive wheeled mobile manipulators following the desired trajectory. The resulting closed-loop system is bounded in probability and the effect due to the external disturbance on the tracking error can be attenuated to any pre-assigned level. Moreover, unknown upper bounds of dynamics uncertainties and disturbances can be estimated by adaptive updated law. The mechanical system with matched disturbances and Markov jumping is solved in terms of a finite set of coupled LMIs. It has been shown that the adaptive control problem for the Markovian jump nonlinear systems is solvable if a set of coupled LMIs have solutions. Finally, a numerical example is given to show the potential of the proposed techniques.

The main contributions of this paper lie in:

(i) developing a reduced model for mobile manipulators such that it could be transformed into the framework of MJLS with modeling system dynamics uncertainties;

(ii) designing an adaptive sliding mode control (SMC) for wheeled mobile manipulators with hybrid joints with Markovian switching;

(iii) the system with matched disturbances and Markov jumping is solved in terms of a finite set of coupled LMIs.

\section{Preliminary}

Lemma 2.1 (see [38]). Let $e=H(s) r$ with $H(s)$ representing an $(n \times m)$-dimensional strictly proper exponentially stable transfer function, $r$ and e denoting its input and output, respectively. Then $r \in L_{2}^{m} \cap L_{\infty}^{m}$ implies that $e, \dot{e} \in L_{2}^{n} \cap L_{\infty}^{n}$, e is continuous, and $e \rightarrow 0$ as $t \rightarrow \infty$. If, in addition, $r \rightarrow 0$ as $t \rightarrow \infty$, then $\dot{e} \rightarrow 0$.

Lemma 2.2 (see [39]). For the matrix $A$ and $B$ with appropriate dimensions, if $(I+A B)$ is nonsingular, then $(I+A B)^{-1}=I-A(I+B A)^{-1} B$.

Theorem 2.3. Given a Markov jump linear system with the system parameter matrices $A_{i}, B_{i}, C_{i}$, $D_{i}$, and $I>\eta^{2} D_{i}^{T} D_{i}$, for $\eta \geq 0, \Phi(t)$ is unknown but satisfying $\|\Phi(t)\| \leq \eta$,

$$
\dot{x}=\left[A_{i}+B_{i}\left[I-\Phi(t) D_{i}\right]^{-1} \Phi(t) C_{i}\right] x,
$$


if there exits $P_{i}>0$ satisfies the following inequality for each $i \in S=1,2, \ldots, N$,

$$
\left[\begin{array}{ccc}
P_{i} A_{i}+A_{i}^{T} P_{i}+\sum_{j=1}^{N} \pi_{i j} P_{j} & \eta P_{i} B_{i} & C_{i}^{T} \\
\eta B_{i}^{T} P_{i}^{T} & -I & \eta D_{i}^{T} \\
C_{i} & \eta D_{i} & -I
\end{array}\right]<0
$$

then the system (2.1) is stable in the mean square sense.

Proof. If there exists a positive definite matrix $P_{i}$ satisfying Lyapunov inequality (2.3), then the indefinite system (2.1) is asymptotically stable:

$$
P_{i} A_{i}+A_{i}^{T} P_{i}+P_{i} B_{i}\left[I-\Phi(t) D_{i}\right]^{-1} \Phi(t) C_{i}+\left(A_{i}+B_{i}\left[I-\Phi(t) D_{i}\right]^{-1} \Phi(t) C_{i}\right)^{T} P_{i}^{T}+\sum_{j=0}^{N} \pi_{i j} P_{j}<0
$$

Let $p_{i}=\left[I-\Phi(t) D_{i}\right]^{-1} \Phi(t) C_{i} x$, then $p_{i}$ can be represented as $p_{i}=\Phi(t)\left[C_{i} x+D_{i} p_{i}\right]$. Then, from the inequality $\|\Phi(t)\| \leq \eta$, we can achieve $p_{i}^{T} p_{i} \leq \eta^{2}\left[C_{i} x+D_{i} p_{i}\right]^{T}\left[C_{i} x+D_{i} p_{i}\right]$. Since

$$
\begin{aligned}
2 x^{T} P_{i} B_{i}\left(I-\Phi(t) D_{i}\right)^{-1} \Phi(t) C_{i} x & =2 x^{T} P_{i} B_{i} p_{i} \\
& \leq 2 x^{T} P_{i} B_{i} p_{i}+\left[C_{i} x+D_{i} p_{i}\right]^{T}\left[C_{i} x+D_{i} p_{i}\right]-\eta^{-2} p_{i}^{T} p_{i} \\
& =x^{T} C_{i}^{T} C_{i} x+2 x^{T}\left[P_{i} B_{i}+C_{i}^{T} D_{i}\right] p_{i} \\
& -\eta^{-2} p_{i}^{T}\left[I-\eta^{2} D_{i}^{T} D_{i}\right] p_{i} .
\end{aligned}
$$

Assume that $a_{i}^{T}=x^{T}\left(P_{i} B_{i}+C_{i}^{T} D_{i}\right), b_{i}=p_{i}, W_{i}=\eta^{2}\left(I-\eta^{2} D_{i}^{T} D_{i}\right)^{-1}$, using inequality (2.4) and Lemma 2.1, there is

$$
\begin{aligned}
& 2 x^{T} P_{i} B_{i}\left(I-\Phi(t) D_{i}\right)^{-1} \Phi(t) C_{i} x \\
& \quad \leq x^{T} C_{i}^{T} C_{i} x+\eta^{2} x^{T}\left(P_{i} B_{i}+C_{i}^{T} D_{i}\right)\left(I-\eta^{2} D_{i}^{T} D_{i}\right)^{-1}\left(P_{i} B_{i}+C_{i}^{T} D_{i}\right)^{T} x .
\end{aligned}
$$

If the following inequality stands, then inequality (2.3) holds:

$$
P_{i} A_{i}+A_{i}^{T} P_{i}+\sum_{j=0}^{N} \pi_{i j} P_{j}+C_{i}^{T} C_{i}+\eta^{2}\left(P_{i} B_{i}+C_{i}^{T} D_{i}\right)\left(I-\eta^{2} D_{i}^{T} D_{i}\right)^{-1}\left(P_{i} B_{i}+C_{i}^{T} D_{i}\right)^{T}<0 .
$$

With Schur Complement, it is easy to transfer (2.6) into (2.2), namely, the system (2.1) is stable. The proof is completed. 
Definition 2.4. A stochastic process $v(t)$ is said to be bounded in probability if the random variables $|v(t)|$ are bounded in probability uniformly in $t$, that is,

$$
\limsup _{r \rightarrow \infty} P|v(t)|>r=0
$$

\section{System Description}

\subsection{Dynamics}

Consider an $n_{a}$ DOF robotic manipulator mounted on a two-wheeled driven mobile platform, the dynamics can be described as:

$$
M(q) \ddot{q}+V(q, \dot{q}) \dot{q}+G(q)+d(t)=B(q) \tau+f,
$$

where $q=\left[q_{v}^{T}, q_{a}^{T}\right]^{T} \in \mathbb{R}^{n}$ with $q_{v}=[x, y, \vartheta]^{T} \in \mathbb{R}^{n_{v}}$ denoting the generalized coordinates for the mobile platform and $q_{a} \in \mathbb{R}^{n_{a}}$ denoting the coordinates of the robotic manipulator joints, and $n=n_{v}+n_{a}$. The symmetric positive definite inertia matrix $M(q) \in \mathbb{R}^{n \times n}$, the Centripetal and Coriolis torques $V(q, \dot{q}) \in \mathbb{R}^{n \times n}$, the gravitational torque vector $G(q) \in \mathbb{R}^{n}$, the known input transformation matrix $B(q) \in \mathbb{R}^{n \times m}$, the control inputs $\tau \in \mathbb{R}^{m}$, and the generalized constraint forces $f \in \mathbb{R}^{n}$ could be represented as, respectively,

$$
\begin{array}{cc}
M(q)=\left[\begin{array}{cc}
M_{v} & M_{v a} \\
M_{a v} & M_{a}
\end{array}\right], \quad V(q, \dot{q})=\left[\begin{array}{cc}
V_{v} & V_{v a} \\
V_{a v} & V_{a}
\end{array}\right], \quad f=\left[\begin{array}{c}
J_{v}^{T} \lambda_{n} \\
0
\end{array}\right], \\
G(q)=\left[\begin{array}{l}
G_{v} \\
G_{a}
\end{array}\right], \quad B(q) \tau=\left[\begin{array}{c}
\tau_{v} \\
\tau_{a}
\end{array}\right], \quad d(t)=\left[\begin{array}{l}
d_{v} \\
d_{a}
\end{array}\right],
\end{array}
$$

where $M_{v}$ and $M_{a}$ describe the inertia matrices for the mobile platform and the links, respectively, $M_{v a}$ and $M_{a v}$ are the coupling inertia matrices of the mobile platform and the links; $V_{v}, V_{a}$ denote the Centripetal and Coriolis torques for the mobile platform, the links, respectively; $V_{v a}, V_{a v}$ are the coupling Centripetal and Coriolis torques of the mobile platform, the links. $G_{v}$ and $G_{a}$ are the gravitational torque vectors for the mobile platform, the links, respectively; $\tau_{v}$ is the input vector associated with the left driven wheel and the right driven wheel, respectively; and $\tau_{a}$ are the control input vectors for the joints of the manipulator; $d_{v}, d_{a}$ denote the external disturbances on the mobile platform, the links, respectively; $J_{v} \in \mathbb{R}^{l \times n_{v}}$ is the kinematic constraint matrix related to nonholonomic constraints; $\lambda_{n} \in \mathbb{R}^{l}$ is the associated Lagrangian multipliers with the generalized nonholonomic constraints. We assume that the mobile manipulator is subject to known nonholonomic constraints. A method of modeling the dynamics of wheeled robots considering wheel-soil interaction mechanics is presented in [40, 41]. For the reason of simplification, we can adopt the methods of producing enough friction between the wheels of the mobile platform and the ground. 


\subsection{Reduced System}

When the system is subjected to nonholonomic constraints, the $(n-m)$ nonintegrable and independent velocity constraints can be expressed as

$$
J_{v}(q) \dot{q}_{v}=0
$$

The constraint (3.3) is referred to as the classical nonholonomic constraint when it is not integrable. In the paper, constraint (3.3) is assumed to be completely nonholonomic and exactly known.

Since $J_{v}(q) \in \mathbb{R}^{\left(n_{v}-m\right) \times n}$ introduce $J_{a} \in \mathbb{R}^{n_{\alpha} \times n}$, and $J=\left[J_{v}, J_{a}\right]^{T} \in \mathbb{R}^{(n-m) \times n}$, such that it is possible to find a $m+n_{a}$ rank matrix $R(q) \in \mathbb{R}^{n \times\left(m+n_{a}\right)}$ formed by a set of smooth and linearly independent vector fields spanning the null space of $J(q)$, that is,

$$
R^{T}(q) J^{T}(q)=0
$$

where $R(q)=\left[r_{1}(q), \ldots, r_{m}(q), r_{m+1}(q), \ldots, r_{m+n_{a}}(q)\right]$. Define an auxiliary time function $\dot{z}(t) \in$ $\mathbb{R}^{m+n_{a}}$, and $\dot{z}(t)=\left[\dot{z}_{1}(t), \ldots, \dot{z}_{m}(t), \dot{z}_{m+1}(t), \ldots, \dot{z}_{m+n_{a}}(t)\right]^{T}$ such that

$$
\dot{q}=R(q) \dot{z}(t)=r_{1}(q) \dot{z}_{1}(t)+\cdots+r_{m}(q) \dot{z}_{m}(t)+r_{m+1}(q) \dot{z}_{m+1}(t)+\cdots+r_{m+n_{a}}(q) \dot{z}_{m+n_{a}}(t)
$$

Equation (3.5) is the kinematic model for the wheeled inverted pendulums. Usually, $\dot{z}(t)$ has physical meaning, consisting of the angular velocity $\omega$, the linear velocity $v$, and the joint

angle vector $\theta_{a}$, that is, $\dot{z}(t)=\left[\begin{array}{lll}v & \omega & \dot{\theta}_{a}^{T}\end{array}\right]^{T}$. Equation (3.5) describes the kinematic relationship between the motion vector $q$ and the velocity vector $\dot{z}(t)$.

Differentiating (3.5) yields

$$
\ddot{q}=\dot{R}(q) \dot{z}+R(q) \ddot{z} .
$$

From (3.5), $\dot{z}$ can be obtained from $q$ and $\dot{q}$ as

$$
\dot{z}=\left[R^{T}(q) R(q)\right]^{-1} R^{T}(q) \dot{q}
$$

The dynamic equation (3.1), which satisfies the nonholonomic constraint (3.3), can be rewritten in terms of the internal state variable $\dot{z}$ as

$$
M(q) R(q) \ddot{z}+V^{*} \dot{z}+G(q)+d(t)=B(q) \tau+J^{T}(q) \lambda,
$$

with $V^{*}=[M(q) \dot{R}(q)+V(q, \dot{q}) R(q)], \lambda=\left[\lambda_{n}, 0\right]^{T}$. 
Table 1: The modes of operation.

\begin{tabular}{lcccccc}
\hline \multirow{2}{*}{ Mode } & \multicolumn{5}{c}{ The modes of hybrid joints } \\
& Right wheel & Left wheel & Joint 1 & Joint 2 & $\ldots$ & Joint $n_{a}$ \\
\hline 1 & normal & normal & normal & normal & $\ldots$ & normal \\
2 & normal & underactuated & normal & normal & $\ldots$ & normal \\
3 & normal & underactuated & underactuated & normal & $\ldots$ & normal \\
4 & normal & underactuated & normal & underactuated & $\ldots$ & normal \\
$\vdots$ & $\vdots$ & $\vdots$ & $\vdots$ & $\vdots$ & $\vdots$ & $\vdots$ \\
$2^{n_{a}+1}$ & normal & underactuated & underactuated & underactuated & $\ldots$ & underactuated \\
\hline
\end{tabular}

Substituting (3.5) and (3.6) into (3.1), and then premultiplying (3.1) by $R^{T}(q)$, the constraint matrix $J^{T}(q) \lambda$ can be eliminated by virtue of (3.4). As a consequence, we have the transformed nonholonomic system

$$
\mathcal{M}(q) \ddot{z}+\mathcal{U}(q, \dot{q}) \dot{z}+\mathcal{G}(q)+\boldsymbol{\Phi}=\boldsymbol{U}
$$

where $\mathcal{M}(q)=R^{T} M(q) R, \mathcal{U}(q, \dot{q})=R^{T}[M(q) \dot{R}+V(q, \dot{q}) R], \mathcal{G}(q)=R^{T} G(q), \boldsymbol{\Phi}=R^{T} d(t)$, $U=R^{T} B(q) \tau$, which is more appropriate for the controller design as the constraint $\lambda$ has been eliminated from the dynamics.

Remark 3.1. In this paper, we choose $z=\left[\theta_{r} \theta_{l} \theta_{1} \theta_{2}, \ldots, \theta_{n_{a}}\right]^{T}$, where $\theta_{r}, \theta_{l}$ denote the rotation angle of the left wheel and the right wheel of the mobile platform, respectively, and $\theta_{1}, \ldots, \theta_{n_{a}}$ denote the joint angles of the link $1,2, \ldots, n_{a}$, respectively, and $\tau=$ $\left[\tau_{r}, \tau_{l}, \tau_{1}, \ldots, \tau_{n_{a}}\right]$.

Remark 3.2. The total degree of freedom for a two-wheeled driven mobile manipulator is $n_{q}=n_{a}+2$.

\subsection{Switching Dynamics}

The hybrid joint is within each actuator of the wheels and links of the mobile manipulator, such that switching may appear in every joint independently. Since the left wheel and right wheel are symmetric, for simplification, we assume that the switching appears in the left wheel and each joint of the manipulator independently. Therefore, there are $2^{n_{a}+1}$ modes of operation, which are listed in Table 1 depending on which hybrid joint is in the active (actuated) or passive (underactuated) mode.

Let $h_{p}$ be the number of passive hybrid joints that have not already reached their set point in a given instant. If $h_{p}>h_{a}, h_{a}$ passive joints are controlled and grouped in the vector $z_{p} \in \mathbb{R}^{h_{a}}$, the remaining passive hybrid joints, if any, are kept locked by the brakes, and the active joints are grouped in the vector $z_{a} \in \mathbb{R}^{h_{a}}$. If $h_{p}<h_{a}$, the $h_{p}$ passive hybrid joints are controlled applying torques in $h_{a}$ active hybrid joints. In this case, $z_{p} \in \mathbb{R}^{h_{p}}$ and $z_{a} \in$ $\mathbb{R}^{h_{a}}$. The strategy is to control all passive hybrid joints until they reach the desired position, considering the conditions exposed above, and then turn on the clutch. After that, all the active hybrid joints are controlled by themselves as a fully-actuated robot. 
The dynamics (3.9) can be partitioned into two parts, the actuated part and the passive part, represented by " $a$ " and " $p$," respectively. Then we can rewrite the dynamics (3.9) as

$$
\left[\begin{array}{cc}
\mathcal{M}_{a}(\zeta) & \mathcal{M}_{a p}(\zeta) \\
\mathcal{M}_{p a}(\zeta) & \mathcal{M}_{p}(\zeta)
\end{array}\right]\left[\begin{array}{c}
\ddot{z}_{a} \\
\ddot{z}_{p}
\end{array}\right]+\left[\begin{array}{cc}
\mho_{a} & \mho_{a p} \\
\mho_{p a} & \mho_{p}
\end{array}\right]\left[\begin{array}{c}
\dot{z}_{a} \\
\dot{z}_{p}
\end{array}\right]+\left[\begin{array}{c}
\mathcal{G}_{a} \\
\mathcal{G}_{p}
\end{array}\right]+\left[\begin{array}{l}
\Phi_{a}(t) \\
\boldsymbol{\Phi}_{p}(t)
\end{array}\right]=\left[\begin{array}{l}
\mathcal{U}_{a} \\
\mathcal{U}_{p}
\end{array}\right]
$$

where

(i) $\mathcal{M}_{a} \in \mathbb{R}^{h_{a} \times h_{a}}, \mathcal{M}_{p} \in \mathbb{R}^{h_{p} \times h_{p}}$ : the inertia matrices of the actuated parts and the passive parts, respectively;

(ii) $\mathscr{M}_{a p} \in \mathbb{R}^{h_{a} \times h_{p}}, \mathcal{M}_{p a} \in \mathbb{R}^{h_{p} \times h_{a}}$ : the coupling inertia matrices of the actuated parts and the passive parts, respectively;

(iii) $\mho_{a} \in \mathbb{R}^{h_{a} \times h_{a}}, V_{p} \in \mathbb{R}^{h_{p} \times h_{p}}$ : the Centripetal and Coriolis torque matrices of the actuated parts and the passive parts, respectively;

(iv) $\mho_{a p} \in \mathbb{R}^{h_{a} \times h_{p}}, \mho_{p a} \in \mathbb{R}^{h_{p} \times h_{a}}$ : the coupling Centripetal and Coriolis torques of the actuated parts and the passive parts, respectively;

(v) $\mathcal{G}_{a} \in \mathbb{R}^{h_{a}}, \mathcal{G}_{p} \in \mathbb{R}^{h_{p}}$ : the gravitational torque vector for the actuated parts and the passive parts, respectively;

(vi) $\Phi_{a}(t) \in \mathbb{R}^{h_{a}}, \Phi_{p}(t) \in \mathbb{R}^{h_{p}}$ : the bounded external disturbance from the environments on the actuated parts and the passive parts, respectively;

(vii) $\varkappa_{a} \in \mathbb{R}^{h_{a}}$ : the control input torque vector for the actuated parts of the joints;

(viii) $\mathfrak{U}_{p} \in \mathbb{R}^{h_{p}}$ : the control input torque vector for the passive parts of the joints satisfying $u_{p}=0$.

After some simple manipulation, we can further obtain

$$
\mathcal{U}_{a}=\bar{M}(z) \ddot{z}_{p}+\bar{H}(z, \dot{z})+\bar{D}(t)
$$

where

$$
\begin{gathered}
\bar{M}=\mathcal{M}_{a}-\mathcal{M}_{a} \mathcal{M}_{p a}^{-1} \mathcal{M}_{p,} \\
\bar{H}=\bar{V}_{1} \dot{z}_{a}+\bar{V}_{2} \dot{z}_{p}+\mathcal{G}_{a}-\mathcal{M}_{a} \mathcal{M}_{p a}^{-1} \mathcal{G}_{p}, \\
\bar{D}(t)=\Phi_{a}-\mathcal{M}_{a} \mathcal{M}_{p a}^{-1} \Phi_{p}, \\
\bar{V}_{1}=V_{a}-\mathcal{M}_{a} \mathcal{M}_{p a}^{-1} V_{p a}, \\
\bar{V}_{2}=V_{a p}-\mathcal{M}_{a} \mathcal{M}_{p a}^{-1} V_{p} .
\end{gathered}
$$




\section{Control Design}

\subsection{Model-Based Control with Unmodeled Dynamics}

Define the tracking errors as

$$
\begin{aligned}
& e=z_{p}-z_{p d}, \\
& \dot{e}=\dot{z}_{p}-\dot{z}_{p d},
\end{aligned}
$$

where $\ddot{z}_{p d}, \dot{z}_{p d}$ and $z_{p d}$ denote the desired trajectories vectors of passive joint accelerations, velocities, and positions, respectively.

The parameters $\bar{M}, \bar{H}$, and $\bar{D}(t)$ in dynamical model (3.11) are functions of physical parameters of mobile manipulators like links masses, links lengths, moments of inertial, and so on. The precise values of these parameters are difficult to acquire due to measuring errors and environment and payloads variations. Therefore, it is assumed that actual value $\bar{M}, \bar{H}$, and $\bar{D}(t)$ can be separated as nominal parts denoted by $\bar{M}_{0}, \bar{H}_{0}$, and $\bar{D}_{0}(t)$ and uncertain parts denoted by $\Delta \bar{M}, \Delta \bar{H}$, and $\Delta \bar{D}(t)$, respectively. These variables satisfy the following relationships:

$$
\begin{gathered}
\bar{M}=\bar{M}_{0}+\Delta M, \\
\bar{H}=\bar{H}_{0}+\Delta H, \\
\bar{D}=\bar{D}_{0}+\Delta D .
\end{gathered}
$$

Suppose that the dynamical models of robot manipulators are known precisely and unmodeled dynamics are excluded, that is, $\Delta M, \Delta H$, and $\Delta D$ in (3.11) are all zeros. At this time, dynamical models (3.11) can be converted into the following nominal models:

$$
\bar{M}_{0}(z) \ddot{z}_{p}+\bar{H}_{0}(z, \dot{z})+\bar{D}_{0}=\mathfrak{U}_{0}
$$

Consider the control law as

$$
u_{0}=\bar{M}_{0}(z)\left(\ddot{z}_{p d}-K_{v} \dot{e}-K_{p} e\right)+\bar{H}_{0}(z, \dot{z})+\bar{D}_{0}
$$

where $K_{v}$ and $K_{p}$ are positive definite matrices. Substituting (4.4) into (4.3) yields

$$
\ddot{e}+K_{v} \dot{e}+K_{p} e=0 .
$$

From Lemma 2.1, it is obvious that errors $\dot{e}$ and $\ddot{e}$ will asymptotically if proportional gain $K_{p}$ and derivative gain $K_{v}$ are chosen in the favorable situation.

According to (4.4), the proposed control is effective based on the strong assumptions that exact knowledge of robot dynamics is precisely known and unmodeled dynamics has to be ignored, which is difficult to obtain in practices. Therefore, we need to approximate dynamics nonlinear functions. One can imagine that model-based control is used to control 
nominal system and another adaptive based control attaching to model-based control for uncertain system can be designed. In this way, applying (4.4) to original systems (3.11) yields

$$
\begin{gathered}
\ddot{e}+K_{v} \dot{e}+K_{p} e=\Xi, \\
\Xi=-\bar{M}_{0}^{-1}\left(\Delta M \ddot{z}_{p}+\Delta H(z, \dot{z})+\Delta D(t)\right),
\end{gathered}
$$

which $\Xi$ is a function of joint variables, physical parameters, parameters variations, unmodeled dynamics, and so on and denotes the structured uncertainty and unstructured uncertainty.

Up to now, the control objective can be restated as: seek a control law based on nominal parameters and adaptive-based compensator such that joint motions of robotic systems (3.11) can follow desired trajectories. The overall control law can be written as

$$
\mathfrak{U}_{a}=\mathfrak{U}_{0}+\mathfrak{U}_{c}
$$

where $\boldsymbol{U}_{c}$ is an adaptive-based controller serving as a compensator for model-based control and designed later. Using control law (4.8), the closed-loop system becomes:

$$
\ddot{e}+K_{v} \dot{e}+K_{p} e=\bar{M}_{0}^{-1} u_{c}+\Xi
$$

Supposed that the state vector is defined as $x=\left[e^{T}, \dot{e}^{T}\right]^{T}$, the state space equation has form as

$$
\begin{gathered}
\dot{x}=A x+B U, \\
A=\left[\begin{array}{cc}
0 & I \\
-K_{p} & -K_{v}
\end{array}\right], \quad B=\left[\begin{array}{l}
0 \\
I
\end{array}\right], \\
U=\bar{M}_{0}^{-1} u_{c}+\Xi .
\end{gathered}
$$

\subsection{Stochastic Control Design}

Since the hybrid joints can be switched among different modes, considering the Markovian jumping, we can rewrite (4.10) by integrating Markovian jumping parameters as

$$
\dot{x}(t)=A\left(r_{t}\right) x(t)+B\left(r_{t}\right) U,
$$

where $r_{t}=j$, and $j$ is one of the Markovian jumping parameters in the limited set $S=$ $\{1,2, \ldots, N\}$ with the mode transition rate matrix $\Pi=\left(\pi_{j \iota}\right),(k, \iota \in N)$. The jump transition probability can be defined as

$$
P\left(r_{t+\Delta t}=\iota \mid r_{t}=k\right)=\left\{\begin{array}{l}
\pi_{k \iota} \Delta t+o(\Delta t), k \neq \iota, \\
1+\pi_{k k} \Delta t+o(\Delta t), \iota=k,
\end{array}\right.
$$


where $\sum_{l=1, l \neq k}^{N} \pi_{k \iota}=-\pi_{k l}, \pi_{k \iota} \geq 0, \forall \iota, k \in \Omega, \iota \neq k$. Here, $\Delta t>0$ and $\lim _{\Delta t \rightarrow 0} O(\Delta t) / \Delta t=0$. The model of the form (4.12) is a hybrid system in which one state $x(t)$ takes values continuously and another state $r_{t}$, referred to as the mode or operating form, takes values discretely in $S$.

For $V(t, x) \in C^{1}$, let us introduce the weak infinitesimal operator $\mathcal{L V}$ of the process $\left\{x(t), \eta_{t}, t \geq 0\right\}$ at the point $\{t, x, j\}$,

$$
\mathcal{L} V=\frac{\partial V}{\partial t}+\frac{\partial V}{\partial x} \dot{x}(t)+\sum_{k=1}^{N} \pi_{k j} V(x, j)
$$

For each possible value $r_{t}=j, j \in S$, we will denote the system matrices associated with mode $j$ by

$$
A\left(r_{t}\right)=A(j)=A_{j}, \quad B\left(r_{t}\right)=B(j)=B_{j},
$$

where $A_{j}, B_{j}$ are known real constant matrices of appropriate dimensions which describe the nominal system.

Theorem 4.1. If the linear matrix inequalities (4.16) have the solution $X_{j}$ for given $A_{j}, B_{j}, X_{j}>0$, and

$$
\left[\begin{array}{ccc}
\widetilde{B}_{j} & 0 & 0 \\
0 & I & 0 \\
0 & 0 & I
\end{array}\right]^{T}\left[\begin{array}{ccc}
\left(A_{j} X_{j}+X_{j} A_{j}^{T}+\sum_{k=1}^{N} \pi_{k j} X_{j}\right) & * & * \\
\eta & -I & \eta I \\
A_{j} X_{j} & \eta I & -I
\end{array}\right]\left[\begin{array}{ccc}
\widetilde{B}_{j} & 0 & 0 \\
0 & I & 0 \\
0 & 0 & I
\end{array}\right]<0,
$$

and define the sliding surface as

$$
\begin{gathered}
\sigma_{j}=S_{j} x_{j}+\gamma \int_{0}^{t} S_{j} x_{j} d t, \\
S_{j}=\left(B_{j}^{T} X_{j}^{-1} B_{j}\right)^{-1} B_{j}^{T} X_{j}^{-1} .
\end{gathered}
$$

Consider the adaptive control as

$$
u_{c}=-\bar{M}_{0} K \sigma_{j}-\bar{M}_{0}\left(S_{j} A_{j} x_{j}+\gamma S_{j} x_{j}\right)-\bar{M}_{0} \frac{1}{b} \sum_{i=1}^{5} \frac{\sigma_{j} \widehat{c}_{i} \Phi_{i}^{2}}{\left\|\sigma_{j}\right\| \Phi_{i}+\delta}
$$

with the adaptive law

$$
\dot{\widehat{c}}_{i}=-\varpi_{i} \widehat{c}_{i}+\frac{\omega_{i} \Phi_{i}^{2}\left\|\sigma_{j}\right\|^{2}}{\left\|\sigma_{j}\right\| \Phi_{i}+\delta_{i}}, \quad i=1, \ldots, 5
$$


where $\widehat{C}=\left[\widehat{c}_{1}, \ldots, \widehat{c}_{5}\right]^{T}$, and $\Phi=\left[\left\|\ddot{z}_{p r}\right\|, 1,\|\dot{z}\|, 1,\|\dot{z}\|\right]^{T}, \omega_{i}>0, K$ is positive definite, $\delta_{i}>0$ and $\varpi_{i}>0(1 \leq i \leq 5)$ satisfying: $\lim _{t \rightarrow \infty} \delta_{i}(t)=0, \int_{0}^{\infty} \delta_{i}(s) d s=\rho_{i \delta}<\infty, \lim _{t \rightarrow \infty} \varpi_{i}(t)=0$, $\int_{0}^{\infty} \varpi_{i}(s) d s=\rho_{i \varpi}<\infty$ with the constants $\rho_{i \delta}$ and $\rho_{i \varpi}$, and $b$ will be defined later. Then, a stable sliding mode exists from the initial time, and the sliding dynamics is stable.

Proof. Define the transfer matrix $T_{j}$ and the related vector $v$, we have

$$
\begin{gathered}
T_{j}=\left[\begin{array}{c}
\left(\widetilde{B}_{j} X_{j} \widetilde{B}_{j}\right) \tilde{B}_{j}^{T} \\
\left(B_{j}^{T} X_{j}^{-1} B_{j}\right)^{-1} B_{j}^{T} X_{j}^{-1}
\end{array}\right], \\
v=\left[\begin{array}{ll}
v_{1} & v_{2}
\end{array}\right]^{T}=T_{j} x_{j},
\end{gathered}
$$

where $v_{1} \in R^{n-m}$, and $v_{2} \in R^{m}, \widetilde{B}_{j}$ is any basis of the null space of $B_{j}^{T}$, that is, $B_{j}$ is an orthogonal complement of $B_{j}$, Note that given any $B_{j}, \widetilde{B}_{j}$ is not unique. Moreover, $T_{j}^{-1}=$ $\left[X_{j} \widetilde{B}_{j} B_{j}\right]$.

Consider (4.22), it is easy to have

$$
\dot{\sigma}_{j}=\dot{v}_{2}+\gamma v_{2}
$$

From the definition of $\sigma_{j}$, we have

$$
\dot{\sigma}_{j}=S_{j}\left(A_{j} x_{j}+B_{j} U\right)+\gamma S_{j} x_{j}
$$

Consider (4.11) and (4.18), we can rewrite (4.24) as

$$
\dot{\sigma}_{j}=S_{j} A_{j} x_{j}+S_{j} B_{j} \bar{M}_{0}^{-1} u_{c}+S_{j} B_{j} \Xi+\gamma S_{j} x_{j}=S_{j} A_{j} x_{j}+\bar{M}_{0}^{-1} u_{c}+\Xi+\gamma S_{j} x_{j} .
$$

with $S_{j} B_{j}=I$.

Consider (4.7), and $\ddot{z}_{p}=\ddot{z}_{r}+\dot{\sigma}_{j}$ with $\ddot{z}_{r}=\ddot{z}_{p d}-\gamma \mathcal{v}_{2}$ and $\dot{\sigma}_{j}=\dot{v}_{2}+\gamma v_{2}$, we can rewrite it as

$$
\begin{aligned}
\Xi & =-\bar{M}_{0}^{-1}\left(\Delta M \ddot{z}_{p}+\Delta H(z, \dot{z})+\Delta D(t)\right) \\
& =-\bar{M}_{0}^{-1} \Delta M \ddot{z}_{r}-\bar{M}_{0}^{-1} \Delta M \dot{\sigma}_{j}-\bar{M}_{0}^{-1} \Delta H(z, \dot{z})-\bar{M}_{0}^{-1} \Delta D
\end{aligned}
$$

Let $\Gamma=\left(I+\bar{M}_{0}^{-1} \Delta M\right)^{-1}$, then we have

$$
\dot{\sigma}_{j}=\Gamma\left(S_{j} A_{j} x_{j}+\gamma S_{j} x_{j}+\bar{M}_{0}^{-1} u_{c}-\bar{M}_{0}^{-1} \Delta M \ddot{z}_{r}-\bar{M}_{0}^{-1} \Delta H(z, \dot{z})-\bar{M}_{0}^{-1} \Delta D\right) .
$$

Let us consider the Lyapunov function as

$$
V_{1}=\sigma_{j}^{T} \sigma_{j}
$$


Taking the derivative (4.28) and integrating (4.27), we have

$$
\begin{aligned}
\dot{V}_{1} & =\sigma_{j}^{T} \dot{\sigma}_{j}+\dot{\sigma}_{j}^{T} \sigma_{j} \\
& =2 \sigma_{j}^{T} \Gamma\left(S_{j} A_{j} x_{j}+\gamma S_{j} x_{j}+\bar{M}_{0}^{-1} u_{c}-\bar{M}_{0}^{-1} \Delta M \ddot{z}_{r}-\bar{M}_{0}^{-1} \Delta H(z, \dot{z})-\bar{M}_{0}^{-1} \Delta D\right) .
\end{aligned}
$$

Substituting (4.19) into (4.29), we have

$$
\begin{aligned}
\dot{V}_{1}= & -2 \sigma_{j}^{T} \Gamma K \sigma_{j}+2 \sigma_{j}^{T} \Gamma \\
& \times\left(-\frac{1}{b} \sum_{i=1}^{5} \frac{\sigma_{j} \widehat{c}_{i} \Phi_{i}^{2}}{\left\|\sigma_{j}\right\| \Phi_{i}+\delta}-\bar{M}_{0}^{-1} \Delta M \ddot{z}_{r}-\bar{M}_{0}^{-1} \Delta H(z, \dot{z})-\bar{M}_{0}^{-1} \Delta D\right) \\
= & -2 \sigma_{j}^{T} \Gamma K \sigma_{j}-2 \sigma_{j}^{T} \Gamma \frac{1}{b} \sum_{i=1}^{5} \frac{\sigma \widehat{c}_{i} \Phi_{i}^{2}}{\left\|\sigma_{j}\right\| \Phi_{i}+\delta}-2 \sigma_{j}^{T} \Gamma \bar{M}_{0}^{-1} \Delta M \ddot{z}_{r} \\
& -2 \sigma_{j}^{T} \Gamma \bar{M}_{0}^{-1} \Delta H(z, \dot{z})-2 \sigma_{j}^{T} \Gamma \bar{M}_{0}^{-1} \Delta D \\
\leq & -2 \sigma_{j}^{T} \Gamma K \sigma_{j}-2 \Gamma \frac{1}{b} \sum_{i=1}^{5} \frac{\left\|\sigma_{j}\right\|^{2} \widehat{c}_{i} \Phi_{i}^{2}}{\left\|\sigma_{j}\right\| \Phi_{i}+\delta}+2\left\|\sigma_{j}\right\|\|\Gamma\|\left\|\bar{M}_{0}^{-1} \Delta M\right\|\left\|\ddot{z}_{r}\right\| \\
& +2\left\|\sigma_{j}\right\|\|\Gamma\|\left\|\bar{M}_{0}^{-1}\right\|\|\Delta H(z, \dot{z})\|+2\left\|\sigma_{j}\right\|\|\Gamma\|\left\|\bar{M}_{0}^{-1}\right\|\|\Delta D\| .
\end{aligned}
$$

Assumption 4.2. There exist some finite positive constants $c_{i}>0(1 \leq i \leq 5)$ such that $\forall z \in$ $R^{n-l}, \forall \dot{z} \in R^{n-l},\|\Gamma\|\left\|\bar{M}_{0}^{-1} \Delta M\right\| \leq c_{1},\|\Gamma\|\left\|\bar{M}_{0}^{-1}\right\|\|\Delta H(z, \dot{z})\| \leq c_{2}+c_{3}\|\dot{z}\|,\|\Gamma\|\left\|\bar{M}_{0}^{-1}\right\|\|\Delta D\| \leq$ $c_{4}+c_{5}\|\dot{z}\|$.

Remark 4.3. For simplification, we assume that $\Delta M>0$. There exist the minimum and maximum eigenvalues $\lambda_{\min }(\Gamma)$ and $\lambda_{\max }(\Gamma)$, such that for all $x \in R^{\left(n-l-n_{p}\right)}$, there exists the known positive parameter $b$ satisfying $0<b \leq \lambda_{\min }(\Gamma)$, that is, $x^{T} b I x \leq x^{T} \lambda_{\min }(\Gamma) I x$.

Remark 4.4. In reality, these constants $c_{i}, 1 \leq i \leq 5$ cannot be obtained beforehand. Although any fixed large $c_{i}$ can guarantee good performance, it is not practical as large $c_{i}$ imply, in general, high noise amplification and high cost of control. Therefore, it is necessary to develop an adaptive law which can approximate the knowledge of $c_{i}, 1 \leq i \leq 5$.

Choose the Lyapunov function candidate $V_{3}=V_{1}+V_{2}$ with

$$
V_{2}=\tilde{C}^{T} \Omega^{-1} \tilde{C}
$$

where $\tilde{C}=C-\widehat{C}$, and $\Omega=\operatorname{diag}\left[\omega_{i}\right], i=1, \ldots, 5$, therefore, we have

$$
\begin{aligned}
V_{3} \leq & -2 \sigma_{j}^{T} \Gamma K \sigma_{j}-2 \Gamma \frac{1}{b} \sum_{i=1}^{5} \frac{\left\|\sigma_{j}\right\|^{2} \widehat{\mathcal{C}}_{i} \Phi_{i}^{2}}{\left\|\sigma_{j}\right\| \Phi_{i}+\delta}+2\left\|\sigma_{j}\right\|\|\Gamma\|\left\|\bar{M}_{0}^{-1} \Delta M\right\|\left\|\ddot{z}_{r}\right\| \\
& +2\left\|\sigma_{j}\right\|\|\Gamma\|\left\|\bar{M}_{0}^{-1}\right\|\|\Delta H(z, \dot{z})\|+2\left\|\sigma_{j}\right\|\|\Gamma\|\left\|\bar{M}_{0}^{-1}\right\|\|\Delta D\|+2 \dot{\tilde{C}}^{T} \Omega^{-1} \widetilde{C} .
\end{aligned}
$$


Integrating (4.20) into (4.32), we have

$$
\begin{aligned}
V_{3} \leq & -2 \sigma_{j}^{T} \Gamma K \sigma_{j}-2 \Gamma \frac{1}{b} \sum_{i=1}^{5} \frac{\left\|\sigma_{j}\right\|^{2} \widehat{c}_{i} \Phi_{i}^{2}}{\left\|\sigma_{j}\right\| \Phi_{i}+\delta}+2\left\|\sigma_{j}\right\|\|\Gamma\|\left\|\bar{M}_{0}^{-1} \Delta M\right\|\left\|\ddot{z}_{r}\right\| \\
& +2\left\|\sigma_{j}\right\|\|\Gamma\|\left\|\bar{M}_{0}^{-1}\right\|\|\Delta H(z, \dot{z})\|+2\left\|\sigma_{j}\right\|\|\Gamma\|\left\|\bar{M}_{0}^{-1}\right\|\|\Delta D\| \\
& +2 \sum_{i=1}^{5} \widehat{c}_{i}^{T} \varpi_{i} \omega_{i}^{-1} \widetilde{c}_{i}-2 \sum_{i=1}^{5} \frac{\widetilde{c}_{i} \Phi_{i}^{2}\left\|\sigma_{j}\right\|^{2}}{\left\|\sigma_{j}\right\|\left\|\Phi_{i}\right\|+\delta_{i}} \\
\leq & -2 \sigma_{j}^{T} \Gamma K \sigma_{j}+2 \sum_{i=1}^{5} \widehat{c}_{i}^{T} \varpi_{i} \omega_{i}^{-1} \widetilde{c}_{i}+\sum_{i=1}^{5} 2 \delta_{i} \\
\leq & -2 \lambda_{\min }(\Gamma K)\left\|\sigma_{j}\right\|^{2}+\sum_{i=1}^{5} \frac{\varpi_{i}}{2 \omega_{i}} c_{i}^{2}+\sum_{i=1}^{5} 2 \delta_{i}
\end{aligned}
$$

with $\widetilde{c}_{i} \widehat{c}_{i}=-\left(\widehat{c}_{i}-(1 / 2) c_{i}\right)^{2}+(1 / 4) c_{i}^{2}$. Therefore, $\dot{V}_{3} \leq-\lambda_{\min }(\Gamma K)\left\|\sigma_{j}\right\|^{2}+\sum_{i=1}^{5}\left(\varpi_{i} / 2 \omega_{i}\right) c_{i}^{2}+$ $\sum_{i=1}^{5} 2 \delta_{i}$. Since $\sum_{i=1}^{5}\left(\varpi_{i} / 2 \omega_{i}\right) c_{i}^{2}+\sum_{i=1}^{5} 2 \delta_{i}$ is bounded, there exists $t>t_{1}, \sum_{i=1}^{5}\left(\varpi_{i} / 2 \omega_{i}\right) c_{i}^{2}+$ $\sum_{i=1}^{5} 2 \delta_{i} \leq \rho_{1}$ with the finite constant $\rho_{1}$, when $\left\|\sigma_{j}\right\| \geq \sqrt{\rho_{1} / \lambda_{\min }(\Gamma K)}$, then $\dot{V}_{3} \leq 0$. For $\left\|\sigma_{j}\right\| \geq$ $\sqrt{\rho_{1} / \lambda_{\min }(\Gamma K)}$, and $\sigma_{j}$ will converge to a compact set denoted by

$$
\Upsilon_{j}:=\left\{\sigma_{j}:\left|\sigma_{j}\right| \leq \sqrt{\frac{\rho_{1}}{\lambda_{\min }(\Gamma K)}}\right\}
$$

From all the above, $\sigma_{j}$ converges to a small set containing the origin as $t \rightarrow \infty$. Moreover, $\sigma_{j} \rightarrow 0$ as $t \rightarrow \infty$ because of $\lim _{t \rightarrow \infty} \delta_{i}(t)=0, \lim _{t \rightarrow \infty} \varpi_{i}(t)=0$, therefore, $\Upsilon_{j}$ converges to the origin, there $\sigma_{j} \rightarrow 0$, therefore, $\dot{v}_{2} \rightarrow 0$ and $v_{2} \rightarrow 0$.

Consider (4.21) and (4.22), we have

$$
\dot{v}=T_{j} \dot{x}_{j}
$$

Consider Theorem 2.3 and (4.23), and let $\dot{\sigma}=0$, it is easy to have

$$
\dot{v}=\left[\begin{array}{c}
\dot{v}_{1} \\
\dot{v}_{2}
\end{array}\right]=\bar{A}_{j} v
$$

where

$$
\bar{A}_{j}=\left[\begin{array}{cc}
\Lambda_{j 1} & \Lambda_{j 2} \\
0 & -\gamma I
\end{array}\right]=\left[\begin{array}{c}
\left(\widetilde{B}_{j}^{T} X \widetilde{B}_{j}\right)^{-1} \widetilde{B}_{j}^{T} A_{j} X_{j} \widetilde{B} \\
0
\end{array}\right.
$$

Therefore, we can partition the state equation as as

$$
\begin{gathered}
\dot{v}_{1}=\Lambda_{j 1} v_{1}+\Lambda_{j 2} v_{2}, \\
\dot{v}_{2}=-\gamma v_{2},
\end{gathered}
$$


Since $v_{2} \rightarrow 0$ and $\dot{v}_{2} \rightarrow 0$, we only consider the stability of $\dot{v}_{1}=\Lambda_{j 1} v_{1}$. It is easy to obtain that if there exists a positive-define matrix $P_{j}=\widetilde{B}_{j}^{T} X_{j} \widetilde{B}_{j}$ enabling the following inequality to hold:

$$
\left[\begin{array}{ccc}
P_{j} \Lambda_{j 1}+\Lambda_{j 1}^{T} P_{j}+\sum_{k=1}^{N} \pi_{k j} P_{j} & * & * \\
\eta \widetilde{B}_{j} & -I & * \\
C_{j} & \eta I & -I
\end{array}\right]<0
$$

where $C_{j}=A_{j} X_{j} \widetilde{B}_{j}$, then the system

$$
\dot{v}_{1}=\Lambda_{j 1} v_{1}
$$

is asymptotically stable, where $X$ is a solution matrix to the LMIs (4.16), which implies that the sliding-mode dynamics (4.36) is asymptotically stable. This implies that (4.39) holds if the matrix inequality shown in (4.16) holds.

Remark 4.5. Note that Theorem 4.1 provides a solution to the problem of adaptive control for mechanical nonlinear systems with Markovian jump parameters. It is worth mentioning that the work conducted in this paper is the attempt to overcome the dynamics uncertainty arising in the sliding mode control for dynamics nonlinear systems with Markovian jump parameters and adopt adaptive control for dynamics nonlinear systems with Markovian jump parameters. The results obtained could be extended to general dynamics systems.

\subsection{Switching Stability}

For the system switching stability between the two different modes, we give the following theorems.

Theorem 4.6. Consider the switching system (4.13) if the system is both stable before and after the switching phase using the control law (4.19). Assume that there exists no external impacts during the switching, the system is also stable during the switching phase.

Proof. Since $\mathbb{V}_{1}$ and $\mathbb{V}_{2}$ are decreasing from Theorem 4.1, we know the system is stable no matter the hybrid joint is either actuated or underactuated. In the preceding, we have shown that the Lyapunov function is nonincreasing during the switching. Let $\mathbb{V}_{12}^{-}=(1 / 2)\left(\dot{\zeta}^{-}-\right.$ $\dot{\zeta}) \Phi\left(\dot{\zeta}^{-}-\dot{\zeta}\right)$ and $\mathbb{V}_{12}^{+}=(1 / 2)\left(\dot{\zeta}^{+}-\dot{\zeta}\right) \Phi\left(\dot{\zeta}^{+}-\dot{\zeta}\right)$ denote the Lyapunov function before and after the switching, and $\zeta^{+}$and $\zeta^{-}$represent the post- and preswitch velocities, respectively. The Lyapunov function change during the switching can be simplified as follows:

$$
\Delta \mathbb{V}=\mathbb{V}^{+}-\mathbb{V}^{-}=\frac{1}{2}\left(\dot{\zeta}^{+}-\dot{\zeta}\right) \Phi\left(\dot{\zeta}^{+}-\dot{\zeta}\right)-\frac{1}{2}\left(\dot{\zeta}^{-}-\dot{\zeta}\right) \Phi\left(\dot{\zeta}^{-}-\dot{\zeta}\right)
$$

There is no external impact during the switching, which means that there are no extra energy injected into the system. Since the inertia properties of the switching joint and link exist, 


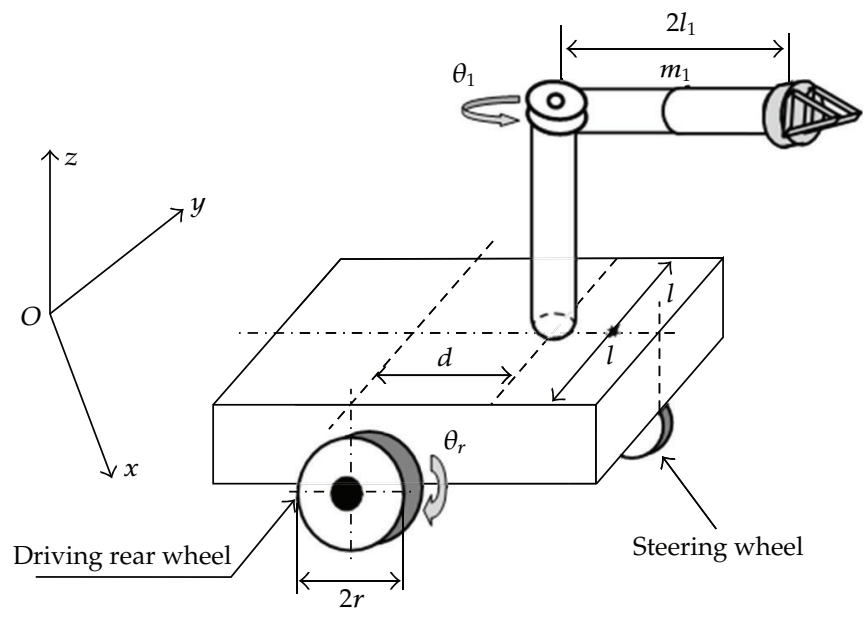

Figure 2: The wheeled mobile manipulator in the simulation.

during the switching joint, if the switching joint is switched from the active mode to the passive mode without considering the friction, the motion of the link should be continuous, that is, $\dot{\zeta}^{+}=\dot{\zeta}^{-}=\dot{\zeta}$. Therefore, during the switching, the Lyapunov function is nonincreasing. If considering the friction, the Lyapunov function is decreasing, that is, $\Delta \mathbb{V} \leq 0$, the motion is stable during the switching. Similarly, if the switching joint is switched from the passive mode to the active mode, although the joint torque is added, since the motion of the system is continuous because of the inertia, that is, $\Delta \mathbb{V} \leq 0$, the motion of the system is also stable.

\section{Simulation Studies}

To verify the effectiveness of the proposed control algorithm, let us consider a wheeled mobile underactuated manipulator shown in Figure 2.

The following variables have been chosen to describe the vehicle (see also Figure 2),

(i) $\tau_{l}, \tau_{r}$ : the torques of two wheels;

(ii) $\tau_{1}$ : the torques of joint 1 ;

(iii) $\theta_{l}, \theta_{r}$ : the rotation angle of the left wheel and the right wheel of the mobile platform;

(iv) $v$ : the forward velocity of the mobile platform;

(v) $\theta$ : the direction angle of the mobile platform;

(vi) $\omega$ : the rotation velocity of the mobile platform, and $\omega=\dot{\theta}$;

(vii) $\theta_{1}$ : the joint angle of the underactuated link;

(viii) $m_{1}, I_{1}, l_{1}$ : the mass, the inertia moment, and the length for the link;

(ix) $r$ : the radius of the wheels;

(x) $l$ : the distance of the wheels;

(xi) $l_{G}$ : the distance between the wheel and joint 1 ;

(xii) $m$ : the mass of the mobile platform;

(xiii) $I$ : the inertia moment of the mobile platform; 
(xiv) $I_{w}$ : the inertia moment of each wheel;

(xv) g: gravity acceleration.

The mobile underactuated manipulator is subject to the following constraint: $\dot{x} \cos \theta-$ $\dot{y} \sin \theta+\dot{\theta} l_{G}=0$. Using the Lagrangian approach, we can obtain the dynamic model with $q=\left[\theta_{l}, \theta_{r}, \theta_{1}\right]^{T}$, then we could obtain

$$
\begin{gathered}
M(q) \ddot{q}+C(q, \dot{q}) \dot{q}+G(q)=B \tau, \\
M(q)=\left[\begin{array}{ccc}
m_{11}(q) & m_{12}(q) & m_{13}(q) \\
* & m_{22}(q) & m_{23}(q) \\
* & * & m_{33}(q)
\end{array}\right], \quad C(q, \dot{q})=\left[\begin{array}{l}
c_{1}(q, \dot{q}) \\
c_{2}(q, \dot{q}) \\
c_{3}(q, \dot{q})
\end{array}\right], \\
B=\left[\begin{array}{lll}
1 & 0 & 0 \\
0 & 1 & 0 \\
0 & 0 & 1
\end{array}\right],
\end{gathered}
$$

where

$$
\begin{gathered}
m_{11}(q)=2 p_{1}+\frac{2 p_{3}}{l^{2}} \tan ^{2} \theta_{r}, \\
m_{12}(q)=\frac{p_{4}}{l} \tan \theta_{r}-p_{8} \sin \theta_{l}, \\
m_{13}(q)=0, \quad m_{22}(q)=2 p_{2}, \\
m_{23}(q)=p_{6}, \quad m_{33}(q)=2 p_{5}, \\
c_{1}(q, \dot{q})=\frac{4 p_{3}}{l^{2}} \tan \theta_{r} \sec ^{2} \theta_{r} \dot{\theta}_{r}^{2} \dot{\theta}_{l}+\frac{p_{4}}{l} \sec \theta_{r} \dot{\theta}_{r}^{2}-2 p_{7} \sec \theta_{r} \sin \theta_{1} \dot{\theta}_{l} \dot{\theta}_{r}^{2} \\
-2 p_{7} \sec ^{2} \theta_{r} \cos \theta_{1} \dot{\theta}_{1} \dot{\theta}_{l}-p_{8} \cos \theta_{1} \dot{\theta}_{1} \dot{\theta}_{r}, \\
c_{2}(q, \dot{q})=\frac{p_{4}}{l} \sec ^{2} \theta_{r} \dot{\theta}_{l} \theta_{r}-p_{8} \cos \theta_{1} \theta_{l} \theta_{1}, \\
p_{1}=\frac{1}{2}\left(m+m_{1}+\frac{I_{w}}{r^{2}}\right), \quad p_{2}=\frac{1}{2}\left(I+I_{m}+m_{1} l_{1}^{2}+I_{1}\right), \quad p_{3}=\frac{1}{2}\left(I_{m}+I_{w}\right), \\
p_{4}=I_{m}, \quad p_{5}=\frac{1}{2}\left(m_{1} l_{1}^{2}+I_{1}\right), \quad p_{6}=m_{1} l_{1}^{2}+I_{1}, \quad p_{7}=\frac{m_{1} l_{1}}{l_{1}}, \quad p_{8}=m_{1} l_{1} .
\end{gathered}
$$

As discussed in Section 2, we set the fully operational configuration represented by $\mathrm{OOO}$ while three possible configurations can occur: $A A P, A P A$, and $A P P$, where $A$ represents actuated joints and $P$ represents passive joints. For example, if we find that a switching occurs in $\tau_{\theta_{1}}$, then the switching configuration to validate the proposed methodology is the $A A P$ configuration. We consider a workspace with a positioning domain which range from $-8^{\circ}$ to $12^{\circ}$, with the velocities set to $1^{\circ} / \mathrm{s}$, and use 2 sectors of position in each joint, denoted as $\mathrm{I}\left(-8^{\circ}: 2^{\circ}\right)$ and II $\left(2^{\circ}: 12^{\circ}\right)$, to map the mobile manipulator workspace. The linearization points with respect to I and II are chosen as $-3^{\circ}$ and $7^{\circ}$, respectively. Then, according to Section 3.2, 8 linearization points with 32 modes are found. For simplification, we select the 8 modes in simulation, which are shown in Table 2. There exist 8 modes for the 
Table 2: Simulation modes.

\begin{tabular}{lcccccc}
\hline \multirow{2}{*}{ Mode } & \multirow{2}{*}{ Joint status } & Mode & Joint status & \multicolumn{3}{c}{ Linearization Section } \\
\hline 1 & $A A A$ & 5 & $A A P$ & $\theta_{r}$ & $\theta_{l}$ & I \\
2 & $A A A$ & 6 & $A A P$ & II & I & I \\
3 & $A P A$ & 7 & $A P P$ & II & I & II \\
4 & $A P A$ & 8 & $A P P$ & II & II & II \\
\hline
\end{tabular}

simulation example, which means an $8 \times 8$ dimension transition rate matrix $\Pi$ is needed, so $\Pi$ is defined as

$$
\Pi=\left[\begin{array}{cccccccc}
-0.72 & 0.15 & 0.22 & 0.21 & 0.14 & 0 & 0 & 0 \\
0.2 & -0.7 & 0.2 & 0.2 & 0 & 0.1 & 0 & 0 \\
0.16 & 0.22 & -0.68 & 0.2 & 0 & 0 & 0.1 & 0 \\
0.22 & 0.3 & 0.2 & -0.82 & 0 & 0 & 0 & 0.1 \\
0 & 0 & 0 & 0 & -0.78 & 0.26 & 0.26 & 0.26 \\
0 & 0 & 0 & 0 & 0.26 & -0.78 & 0.26 & 0.26 \\
0 & 0 & 0 & 0 & 0.26 & 0.26 & -0.78 & 0.26 \\
0 & 0 & 0 & 0 & 0.26 & 0.26 & 0.26 & -0.78
\end{array}\right]
$$

The system parameters are chosen as $G=0 \mathrm{~kg}, B=I, m=10.0 \mathrm{~kg}, m_{1}=2.0 \mathrm{~kg}, I=1.0 \mathrm{~kg} \cdot \mathrm{m}^{2}$, $I_{1}=1.0 \mathrm{~kg} \cdot \mathrm{m}^{2}, I_{m}=2.0 \mathrm{~kg} \cdot \mathrm{m}^{2}, I_{w}=2.0 \mathrm{~kg} \cdot \mathrm{m}^{2}, l=1.0 \mathrm{~m}, l_{1}=1.0 \mathrm{~m}, r=0.5 \mathrm{~m}$.

Assume that the nominal models are obtained as:

$$
\begin{aligned}
& A_{1}=\left[\begin{array}{cccc}
0 & 0 & 1.0000 & 0 \\
0 & 0 & 0 & 1.0000 \\
0.0040 & 0.0012 & 0.0653 & -0.0728 \\
-0.0047 & -0.0010 & -0.0717 & 0.0647
\end{array}\right], \quad B_{1}=\left[\begin{array}{cc}
0 & 0 \\
0 & 0 \\
0.0003 & 0.3354 \\
-0.0003 & -0.0020
\end{array}\right] \text {, } \\
& A_{2}=\left[\begin{array}{cccc}
0 & 0 & 1.0000 & 0 \\
0 & 0 & 0 & 1.0000 \\
0.0040 & 0.0012 & 0.0653 & -0.0728 \\
-0.0047 & -0.0010 & -0.0717 & 0.0647
\end{array}\right], \quad B_{2}=\left[\begin{array}{cc}
0 & 0 \\
0 & 0 \\
0.0003 & 0.3354 \\
-0.0003 & 0.3333
\end{array}\right] \text {, } \\
& A_{3}=\left[\begin{array}{cccc}
0 & 0 & 1.0000 & 0 \\
0 & 0 & 0 & 1.0000 \\
0.0057 & 0.0014 & 0.0725 & -0.0764 \\
-0.0064 & -0.0011 & -0.0790 & 0.0676
\end{array}\right], \quad B_{3}=\left[\begin{array}{cc}
0 & 0 \\
0 & 0 \\
0.0035 & 0.3582 \\
-0.0035 & -0.0249
\end{array}\right] \text {, } \\
& A_{4}=\left[\begin{array}{cccc}
0 & 0 & 1.0000 & 0 \\
0 & 0 & 0 & 1.0000 \\
0.0057 & 0.0014 & 0.0725 & -0.0764 \\
-0.0064 & -0.0011 & -0.0790 & 0.0676
\end{array}\right], \quad B_{4}=\left[\begin{array}{cc}
0 & 0 \\
0 & 0 \\
0.0035 & 0.3582 \\
-0.0035 & 0.3333
\end{array}\right] \text {, }
\end{aligned}
$$


Journal of Applied Mathematics

$$
\begin{aligned}
& A_{5}=\left[\begin{array}{cccc}
0 & 0 & 1.0000 & 0 \\
0 & 0 & 0 & 1.0000 \\
0.0042 & 0.0016 & 0.0628 & -0.0686 \\
-0.0048 & -0.0013 & -0.0691 & 0.0606
\end{array}\right], \quad B_{5}=\left[\begin{array}{cc}
0 & 0 \\
0 & 0 \\
-0.0022 & 0.3175 \\
0.0022 & 0.0158
\end{array}\right] \text {, } \\
& A_{6}=\left[\begin{array}{cccc}
0 & 0 & 1.0000 & 0 \\
0 & 0 & 0 & 1.0000 \\
0.0042 & 0.0016 & 0.0628 & -0.0686 \\
-0.0048 & -0.0013 & -0.0691 & 0.0606
\end{array}\right], \quad B_{6}=\left[\begin{array}{cc}
0 & 0 \\
0 & 0 \\
-0.0022 & 0.3175 \\
0.0022 & 0.3333
\end{array}\right] \text {, } \\
& A_{7}=\left[\begin{array}{cccc}
0 & 0 & 1.0000 & 0 \\
0 & 0 & 0 & 1.0000 \\
0.0055 & 0.0010 & 0.0753 & -0.0809 \\
-0.0062 & -0.0008 & -0.0819 & 0.0719
\end{array}\right], \quad B_{7}=\left[\begin{array}{cc}
0 & 0 \\
0 & 0 \\
0.0068 & 0.3808 \\
-0.0068 & -0.0475
\end{array}\right] \text {, } \\
& A_{8}=\left[\begin{array}{cccc}
0 & 0 & 1.0000 & 0 \\
0 & 0 & 0 & 1.0000 \\
0.0055 & 0.0010 & 0.0753 & -0.0809 \\
-0.0062 & -0.0008 & -0.0819 & 0.0719
\end{array}\right], \quad B_{8}=\left[\begin{array}{cc}
0 & 0 \\
0 & 0 \\
0.0068 & 0.3808 \\
-0.0068 & 0.3333
\end{array}\right] \text {. }
\end{aligned}
$$

The parameters in (4.19) are set as $C(0)=[0.00002, \ldots, 0.00002]^{T}$, for $i=1,2, \ldots, 8, M_{0}=I$, $\omega_{i}=0.5, \alpha_{i}=\delta=1 /(t+1)^{2}, K=\operatorname{diag}[1.0], b=1.0, \gamma=1.0$. The initial condition we used for simulation is $x_{0}=[0.3,0.3,0.2,-0.1,0.1,-0.15]^{T}$. Via LMI optimization with the data $A_{j}, B_{j}$, we can get the following solution to the LMIs (4.16) as:

$$
\begin{aligned}
& X_{1}=10^{4}\left[\begin{array}{cccc}
1.9625 & 0.0001 & -0.0012 & 0.0011 \\
0.0001 & 1.9620 & -0.0003 & 0.0002 \\
-0.0012 & -0.0003 & 1.0267 & 0.0000 \\
0.0011 & 0.0002 & 0.0000 & 1.0267
\end{array}\right], \\
& X_{2}=10^{3}\left[\begin{array}{cccc}
2.7094 & -0.0148 & -0.0022 & 0.0015 \\
-0.0148 & 2.7654 & -0.0004 & -0.0003 \\
-0.0022 & -0.0004 & 4.8496 & -0.0000 \\
0.0015 & -0.0003 & -0.0000 & 4.8496
\end{array}\right], \\
& X_{3}=10^{3}\left[\begin{array}{cccc}
2.4313 & -0.0153 & -0.0028 & 0.0020 \\
-0.0153 & 2.4918 & -0.0005 & -0.0002 \\
-0.0028 & -0.0005 & 4.8077 & -0.0000 \\
0.0020 & -0.0002 & -0.0000 & 4.8077
\end{array}\right], \\
& X_{4}=10^{3}\left[\begin{array}{cccc}
2.2073 & -0.0145 & -0.0026 & 0.0018 \\
-0.0145 & 2.2639 & -0.0004 & -0.0003 \\
-0.0026 & -0.0004 & 4.7749 & -0.0000 \\
0.0018 & -0.0003 & -0.0000 & 4.7749
\end{array}\right],
\end{aligned}
$$




$$
\begin{aligned}
& X_{5}=10^{3}\left[\begin{array}{cccc}
2.8110 & -0.0160 & -0.0023 & 0.0016 \\
-0.0160 & 2.8679 & -0.0005 & -0.0002 \\
-0.0023 & -0.0005 & 4.8654 & -0.0000 \\
0.0016 & -0.0002 & -0.0000 & 4.8654
\end{array}\right], \\
& X_{6}=10^{3}\left[\begin{array}{cccc}
5.2731 & -0.0149 & -0.0037 & 0.0030 \\
-0.0149 & 5.3153 & -0.0010 & 0.0003 \\
-0.0037 & -0.0010 & 5.3202 & -0.0000 \\
0.0030 & 0.0003 & -0.0000 & 5.3202
\end{array}\right], \\
& X_{7}=10^{3}\left[\begin{array}{cccc}
5.2363 & -0.0123 & -0.0054 & 0.0045 \\
-0.0123 & 5.3133 & -0.0007 & 0.0000 \\
-0.0054 & -0.0007 & 5.3153 & -0.0000 \\
0.0045 & 0.0000 & -0.0000 & 5.3153
\end{array}\right], \\
& X_{8}=\left[\begin{array}{llll}
-0.6928 & -0.0000 & -0.0000 & -0.0000 \\
-0.0000 & -0.6928 & -0.0000 & -0.0000 \\
-0.0000 & -0.0000 & -0.6928 & -0.0000 \\
-0.0000 & -0.0000 & -0.0000 & -0.6928
\end{array}\right] .
\end{aligned}
$$

So we can obtain the solution of $S_{i}$, for $i=1,2, \ldots, 8$. Torque disturbances $D(t)$ are introduced to verify the robustness of the controllers

$$
\left[\begin{array}{l}
d_{r}(t) \\
d_{l}(t) \\
d_{1}(t)
\end{array}\right]=\left[\begin{array}{c}
0.023 \sin (4 t) \\
0.007 \sin (3 t)+0.009 \cos ^{2} t \\
0.015 \cos (5 t)
\end{array}\right]
$$

The disturbance is turned off after the switching introduction in corresponding joint or wheel.

The system switches among the 8 modes randomly during operation. From Figure 3, we can see that firstly the system switches from mode 1 to mode 4 , then from mode 4 to mode 1, finally, it switches from mode 1 to modes 4,6 , and 7 . Figure 4 shows that the system is stabilized during operation. From Figures 5, 6, and 7, it can be noticed that the torque inputs are bounded. The simulation results demonstrate the tracking error decays to the equilibrium point under the designed mode-dependent controller.

\section{Conclusion}

In this paper, we consider stochastic stability and sliding mode control for mobile manipulators using stochastic jumps switching joints. Adaptive parameter techniques are adopted to cope with the effect of the Markovian switching and nonlinear dynamics uncertainty and follow the desired trajectory for wheeled mobile manipulators. The resulting closed-loop system is bounded in probability and the effect due to the external disturbance on the tracking errors can be attenuated to any preassigned level. It has been shown that the adaptive control problem for the Markovian jump nonlinear systems is solvable if a set of coupled LMIs have solutions. Finally, a numerical example is given to show the potential of the proposed techniques. 


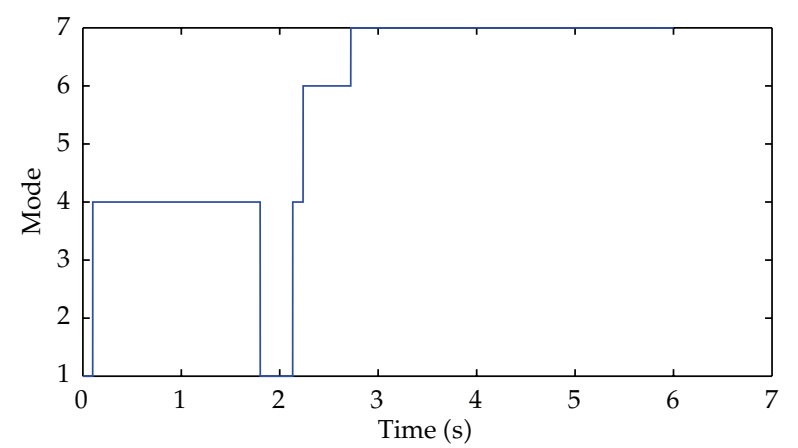

Figure 3: Mode jumping.

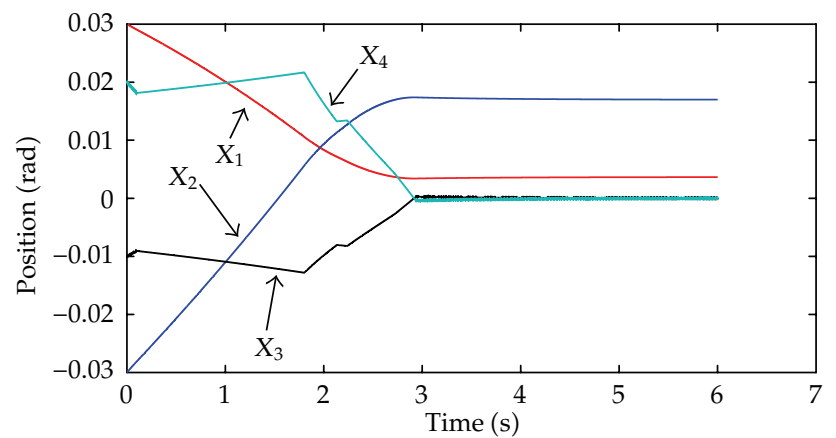

Figure 4: States.

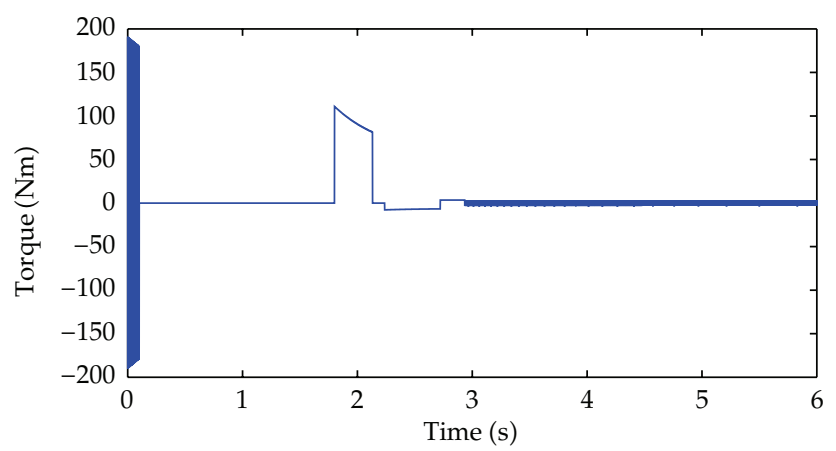

Figure 5: The input control $u_{1}$.

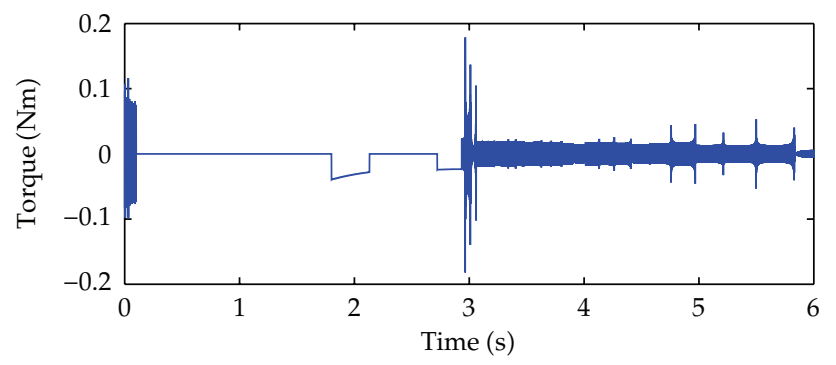

Figure 6: The input control $u_{2}$. 


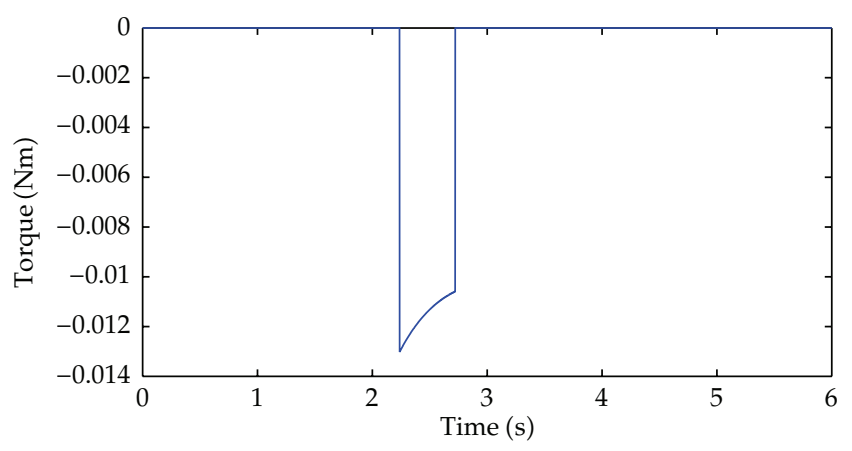

Figure 7: The input control $u_{3}$.

\section{Acknowledgment}

This study was partially supported by the National Natural Science Foundation of China (Grant no. 61005080), Special Postdoctoral Foundation of China (Grant no. 201104405), Postdoctoral Foundation of China (20100480994), and the "111" Project (B07018). The authors would like to express their acknowledgment to Dr. Z. J. Li for his help in theory and literature.

\section{References}

[1] Z. Li, A. Ming, N. Xi, and M. Shimojo, "Motion control of nonholonomic mobile underactuated manipulator," in Proceedings of the IEEE International Conference on Robotics and Automation, pp. 3512 3519, May 2006.

[2] Z. Li, A. Ming, N. Xi, J. Gu, and M. Shimojo, "Development of hybrid joints for the complaint arm of human-symbiotic mobile manipulator," International Journal of Robotics and Automation, vol. 20, no. 4, pp. 260-270, 2005.

[3] Z. Li, C. Yang, J. Luo, Z. Wang, and A. Ming, "Robust motion/force control of nonholonomic mobile manipulatorsusing hybrid joints," Advanced Robotics, vol. 21, no. 11, pp. 1231-1252, 2007.

[4] Z. Li and Y. Kang, "Dynamic coupling switching control incorporating support vector machines for wheeled mobile manipulators with hybrid joints," Automatica, vol. 46, no. 5, pp. 785-958, 2010.

[5] Z. Li, Y. Yang, and J. Li, "Adaptive motion/force control of mobile under-actuated manipulators with dynamics uncertainties by dynamic coupling and output feedback," IEEE Transactions Control System Technology, vol. 18, no. 5, pp. 1068-1079, 2010.

[6] S. S. Ge and Z. Li, "Data driven adaptive predictive control for holonomic constrained under-actuated biped robots," IEEE Transactions on Control Systems Technology, vol. 20, no. 3, pp. 787-795, 2012.

[7] S. Lin and A. A. Goldenberg, "Neural-network control of mobile manipulators," IEEE Transaction on Neural Network, vol. 12, no. 5, pp. 1121-1133, 2001.

[8] Z. Li, S. S. Ge, and A. Ming, "Adaptive robust motion/force control of holonomic-constrained nonholonomic mobile manipulator," IEEE Transactions System, Man, and Cybernetics B, vol. 37, no. 3, pp. 607-617, 2007.

[9] Z. Li, J. Li, and Y. kang, "Adaptive robust coordinated control of multiple mobile manipulators interacting with rigid environments," Automatica, vol. 46, pp. 2028-2034, 2010.

[10] W. Dong, "On trajectory and force tracking control of constrained mobile manipulators with parameter uncertainty," Automatica, vol. 38, no. 9, pp. 1475-1484, 2002.

[11] Z. Li, P. Tao, S. S. Ge, M. D. Adams, and W. S. Wijesoma, "Robust adaptive control of cooperating mobile manipulators with relative motion," IEEE Transactions System, Man, and Cybernetics B, vol. 39, no. 1, pp. 103-116, 2009.

[12] Z. Li, W. Chen, and J. Luo, "Adaptive compliant force-motion control of coordinated nonholonomic mobile manipulators interacting with unknown non-rigid environments," Neurocomputing, vol. 71, no. 7-9, pp. 1330-1344, 2008. 
[13] Y. Yamamoto and X. Yun, "Unified analysis on mobility and manipulability of mobile manipulators," in Proceedings of the IEEE International Conference on Robotics and Automation, pp. 1200-1206, 1999.

[14] Z. Li, J. Gu, A. Ming, and C. Xu, "Intelligent complaint force/motion control of nonholonomic mobile manipulator working on the non-rigid surface," Neural Computing and Applications, vol. 15, no. 3-4, pp. 204-216, 2006.

[15] Z. Li, C. Yang, and J. Gu, "Neuro-adaptive compliant force/ motion control for uncertain constrained wheeled mobile manipulator," International Journal of Robotics and Automation, vol. 22, no. 3, pp. 206214, 2007.

[16] Z. Li, S. S. Ge, M. Adams, and W. S. Wijesoma, "Robust adaptive control of uncertain force/motion constrained nonholonomic mobile manipulators," Automatica, vol. 44, no. 3, pp. 776-784, 2008.

[17] Y. Liu and Y. Li, "Robust adaptive neuro-fuzzy control for nonholonomic mobile modular manipulats in task space," in Proceedings of the IEEE International Conference on Robotics and Biomimetics, pp. 66-71, 2005.

[18] K. Tchon, "Repeatability of inverse kinematics algorithms for mobile manipulators," Institute of Electrical and Electronics Engineers, vol. 47, no. 8, pp. 1376-1380, 2002.

[19] Z. Li and W. Chen, "Adaptive neural-fuzzy control of uncertain constrained multiple coordinated nonholonomic mobile manipulators," Engineering Applications of Artificial Intelligence, vol. 21, no. 7, pp. 985-1000, 2008.

[20] J. G. Tao, X. Li, F. Yang, and Z. Q. Deng, "A wheel-arm reconfigurable mobile robot design and its reconfigurable configuration," in Proceedings of the ASME/IFToMM International Conference on Reconfigurable Mechanisms and Robots, pp. 550-557, 2008.

[21] Z. Li, S. S. Ge, M. Adams, and W. S. Wijesoma, "Adaptive robust output-feedback motion/force control of electrically driven nonholonomic mobile manipulators," IEEE Transactions on Control Systems Technology, vol. 16, no. 6, pp. 1308-1315, 2008.

[22] Z. Li, S. S. Ge, and Z. Wang, "Robust adaptive control of coordinated multiple mobile manipulators," Mechatronics, vol. 18, pp. 239-250, 2008.

[23] R. Brooks, L. Aryanada, A. Edsinger et al., "Sensing and manipulating built-for-human environments," International Journal of Humanoid Robotics, vol. 1, no. 1, pp. 1-28, 2004.

[24] S. S. Ge, J. Wang, T. H. Lee, and G. Y. Zhou, "Adaptive robust stabilization of dynamic nonholonomic chained systems," Journal of Robotic System, vol. 18, no. 3, pp. 119-133, 2001.

[25] S. S. Ge, Z. Wang, and T. H. Lee, "Adaptive stabilization of uncertain nonholonomic systems by state and output feedback," Automatica, vol. 39, no. 8, pp. 1451-1460, 2003.

[26] A. De Luca and G. Oriolo, "Trajectory planning and control for planar robots with passive last joint," The International Journal of Robotics Research, vol. 21, no. 5-6, pp. 575-590, 2002.

[27] H. Arai and K. Tanie, "Nonholonomic control of a three-DOF planar underactuted manipulator," IEEE Transactions Robotics and Automation, vol. 14, no. 5, pp. 681-694, 1998.

[28] M. Bergerman, C. Lee, and Y. Xu, "A dynamic coupling index for underactuated manipulators," Journal of Robotic Systems, vol. 12, no. 10, pp. 693-707, 1995.

[29] Z. Li, J. Zhang, and Y. Yang, "Motion control of mobile under-actuated manipulators by implicit function using support vector machines," IET Control Theory \& Applications, vol. 4, no. 11, pp. 23562368, 2010.

[30] Z. Li, "Adaptive fuzzy output feedback motion/force control for wheeled inverted pendulums," IET Control Theory \& Applications, vol. 5, no. 10, pp. 1176-1188, 2011.

[31] M. W. Spong, "The swing up control problem for the Acrobot," IEEE Control Systems, vol. 15, pp. 49-55, 1995.

[32] Z. Li, Y. Zhang, and Y. Yang, "Support vector machine optimal control for mobile wheeled inverted pendulums with unmodelled dynamics," Neurocomputing, vol. 73, pp. 2773-2782, 2010.

[33] Z. Li and C. Xu, "Adaptive fuzzy logic control of dynamic balance and motion for wheeled inverted pendulums," Fuzzy Sets and Systems, vol. 160, no. 12, pp. 1787-1803, 2009.

[34] Z. Li and J. Luo, "Adaptive robust dynamic balance and motion controls of mobile wheeled inverted pendulums," IEEE Transactions on Control Systems Technology, vol. 17, no. 1, pp. 233-241, 2009.

[35] Y. Liu, Y. Xu, and M. Bergerman, "Cooperation control of multiple manipulators with passive joints," IEEE Transactions Robotics and Automation, vol. 15, no. 2, pp. 258-267, 1999.

[36] R. Tinos, M. H. Terra, and J. Y. Ishihara, "Motion and force control of cooperative robotic manipulators with passive joints," IEEE Transactions Control Systems Technology, vol. 14, no. 4, pp. 725-734, 2006.

[37] Y. Kang, Z. Li, Y. Dong, and H. Xi, "Markovian based fault-tolerant control for wheeled mobile manipulators," IEEE Transactions Control System Technology, vol. 20, no. 1, pp. 266-276, 2012. 
[38] S. S. Ge, T. H. Lee, and C. J. Harris, Adaptive Neural Network Control of Robot Manipulators, World Scientific, London, UK, 1998.

[39] A. Weinmann, Uncertain Models and Robust Control, Springer, New York, NY, USA, 1991.

[40] L. Ding, K. Nagatani, K. Sato et al., "Terramechanics-based high-fidelity dynamics simulation for wheeled mobile robot on deformable rough terrain," IEEE International Conference on Robotics and Automation, pp. 4922-4927, 2010.

[41] L. Ding, H. Gao, Z. Deng, and W. Li, "Advances in simulation of planetary wheeled mobile robots," in Mobile Robots-Current Trends, chapter 18, pp. 375-402, Intech Press, 2011. 


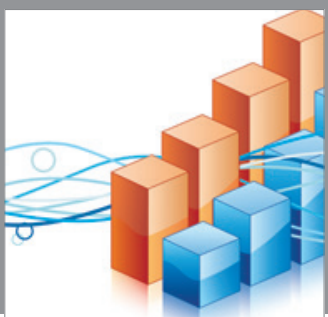

Advances in

Operations Research

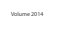

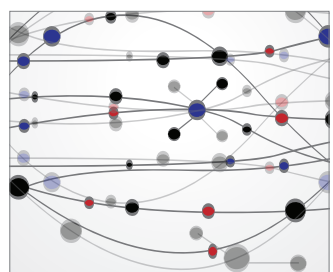

\section{The Scientific} World Journal
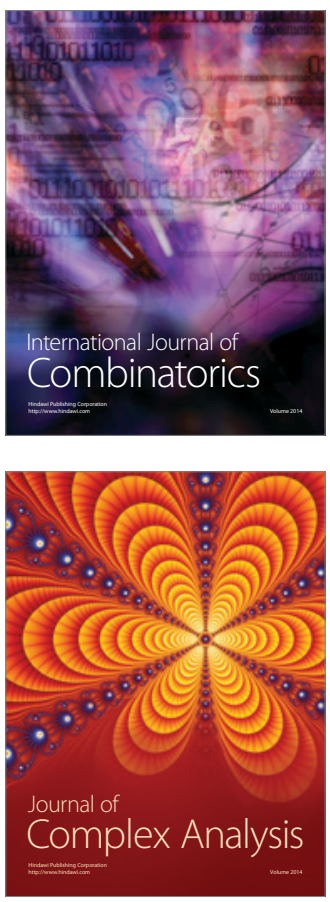

International Journal of

Mathematics and

Mathematical

Sciences
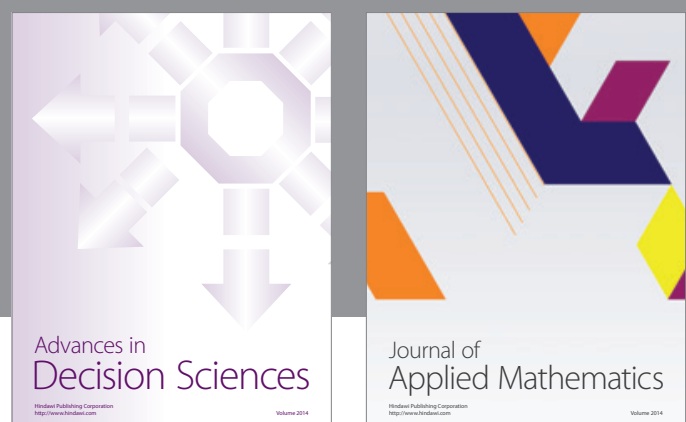

Journal of

Applied Mathematics
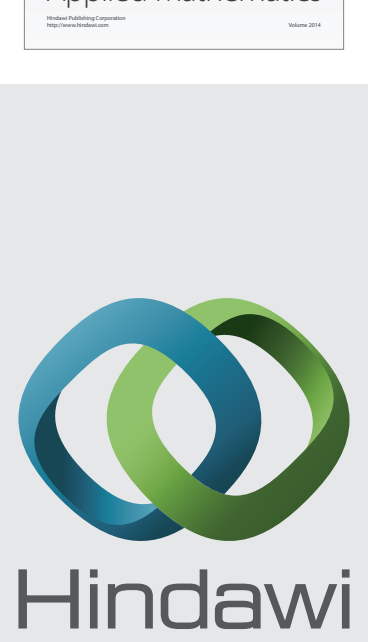

Submit your manuscripts at http://www.hindawi.com
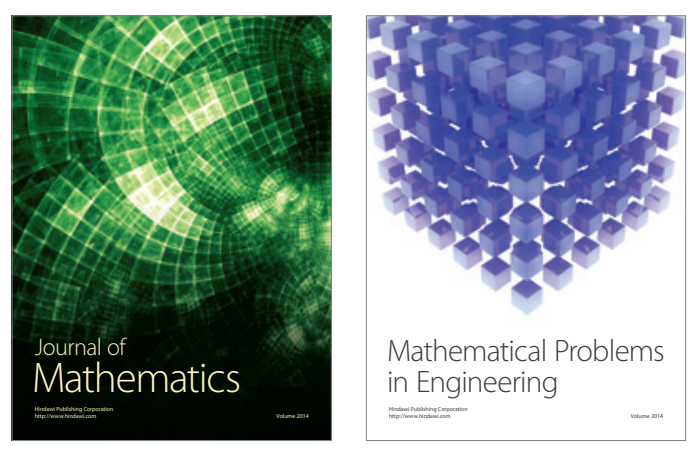

Mathematical Problems in Engineering
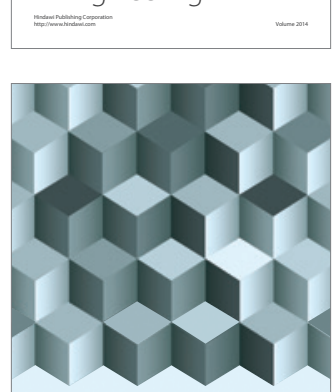

Journal of

Function Spaces
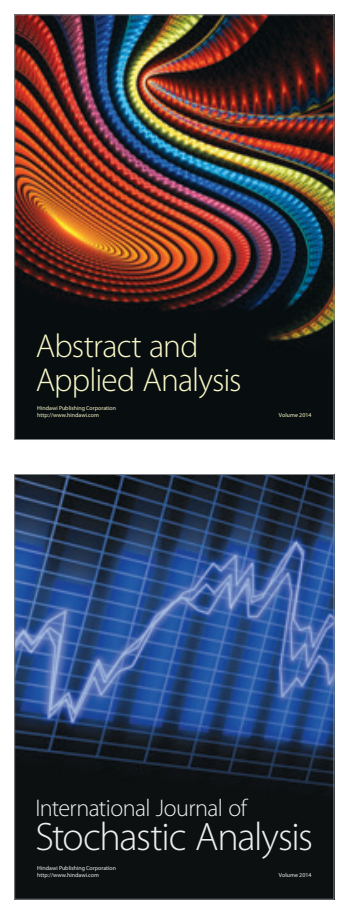

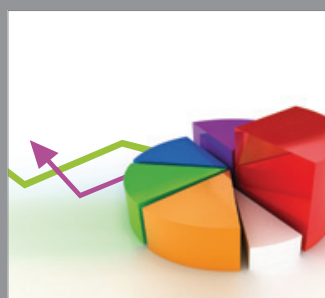

ournal of

Probability and Statistics

Promensencen
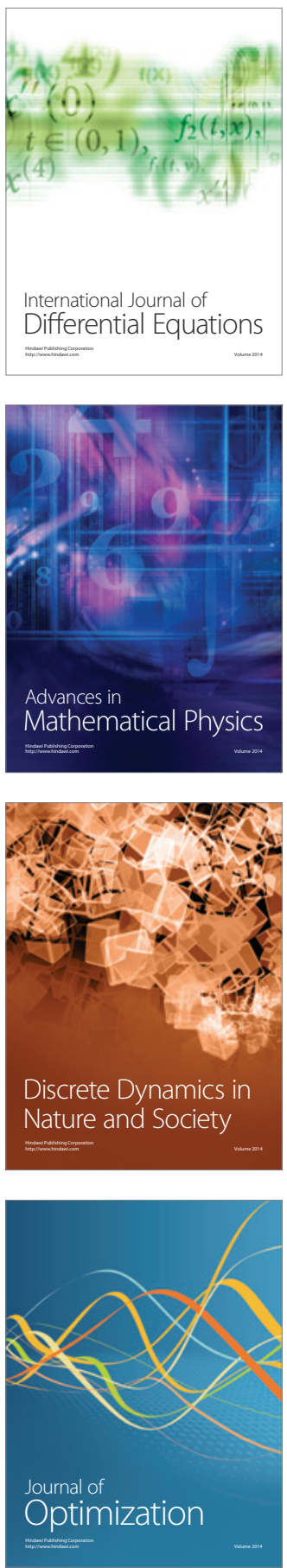